\title{
All Weather Road Edge Identification Based on Driving Video Mining
}

\author{
Zheyuan Wang, Guo Cheng \\ Department of Computer Science, \\ Indiana University Purdue University Indianapolis, USA \\ zheywang@iupui.edu guocheng@iupui.edu
}

\begin{abstract}
- to avoid vehicle running off road, road edge detection is a fundamental function. Current work on road edge detection has not exhaustively tackled all weather and illumination conditions. We first sort the visual appearance of roads based on physical and optical properties under various illuminations. Then, data mining approach is applied to a large driving video set that contains the full spectrum of seasons and weathers to learn the statistical distribution of road edge appearances. The obtained parameters of road environment in color on road structure are used to classify weather in video briefly, and the corresponding algorithm and features are applied for robust road edge detection. To visualize the road appearance as well as evaluate the accuracy of detected road, a compact road profile image is generated to reduce the data to a small fraction of video. Through the exhaustive examination of all weather and illuminations, our road detection methods can locate road edges in good weather, reduce errors in dark illuminations, and report road invisibility in poor illuminations.
\end{abstract}

Keywords - road edge detection; environment perception; weather and illumination; driving video; data mining; autonomous vehicle;

\section{INTRODUCTION}

To guide a vehicle along a road, lane mark tracking has been successfully applied $[3,7,16]$. With unique white patterns painted on road surface, various methods have been able to locate them with a high accuracy. However, vehicles still need to recognize road edges of various materials for the roads without clear lane marks such as community road, rural roads, city roads with only curbs, the roads where lane marks are in worn status, imperfect reflection on wet, snow roads, etc. Although LiDAR can detect 3D targets, they are inherently not more powerful than vision in finding road edges without large elevation difference there $[2,9]$.

A human driver has less difficulty in guiding a vehicle within a road by vision. Even animals like horses are able to run on road. The minimum criteria used for road understanding are: (1) color difference between on-road and off-road because of the material or elevation change, (2) linearity of constructed road edges stretching forward for vehicle passing, (3) homogeneity on road surface due to road pavement. For vision based road detection, various computational methods have been proposed $[3,4,5,6,8,12]$. These algorithms focused on one or several features to detect majority of road, but left a critical issue unsolved. That is how to guarantee the success of road detection in all environments and illuminations, or declare the incapability of detection when visual distinction is weak. There has no systematic collection, analysis and testing of full spectrums of road and illuminations, although effort has been

\author{
Jiang Yu Zheng \\ Department of Computer Science, and TASI \\ Indiana University Purdue University Indianapolis, USA \\ jzheng@iupui.edu
}

made to handle individual types of weathers $[1,14,15,16]$. This makes the algorithms unready for autonomous vehicles, in which every instance is critical for preventing road departure.

Our work here aims at solving the road edge detection by vision inspired by the capability of human drivers. It may enhance the lane mark following in a safer infrastructure, and switch to another sensor like GPS or V2V if road is invisible. The originality of the paper is to learn from data [10] in naturalistic driving video [13] for road edge identification. Two aspects of analysis are given. Qualitatively, we categorize the road appearances under various illuminations based on reflection model [10], in addition to the ad-hoc criteria such as surface homogeneity, edge linearity, and road color properties in previous road edge detection. Quantitatively, we adopt data mining approach to find feature distributions for all weather categories and estimate the best features for road edge identification. In this process, the road profile [11] is the first key element in analysis and visualization because it converts video to a compact image and data sequence, which is required for large video datasets. The detected road edges are displayed in the road profiles for comparison and verification.

In Sec. II, the road profile is first introduced for a large naturalistic driving video set. In Sec. III, different types of weather and illuminations are clustered by data mining algorithm, and video frames are classified. In Sec. IV, the features are selected qualitatively for the identified weathers, and precise road edge detection is carried out based on data mining results. Sec. V gives the experiments and evaluation.

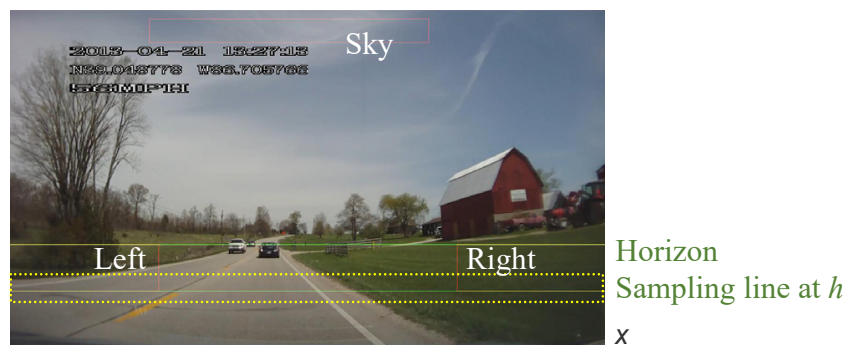

Fig. 1. Sample video frames for a road profile and weather data. Yellow belt around a sampling line is used to calculate the linearity of road edges. Weather sensitive data are sampled in three regions indicated in the image.

\section{ROAD PROFILES FOR DATA CONDENSING}

The driving video is costly in storage, analysis, and test due to its large data volume. We convert a video clip to a temporal image called road profile [11] for data condensing. After the horizon is calibrated in the HD video frame at a fixed height for a vehicle, we set a horizontal pixel line at $h$ pixels below the horizon to sample all video frames taken from that vehicle 
(Fig. 1). The sampling line scans the road about $15 \mathrm{~m}$ ahead considering a necessary time to respond to road departure. The line should not be set too far to capture other vehicles in front.
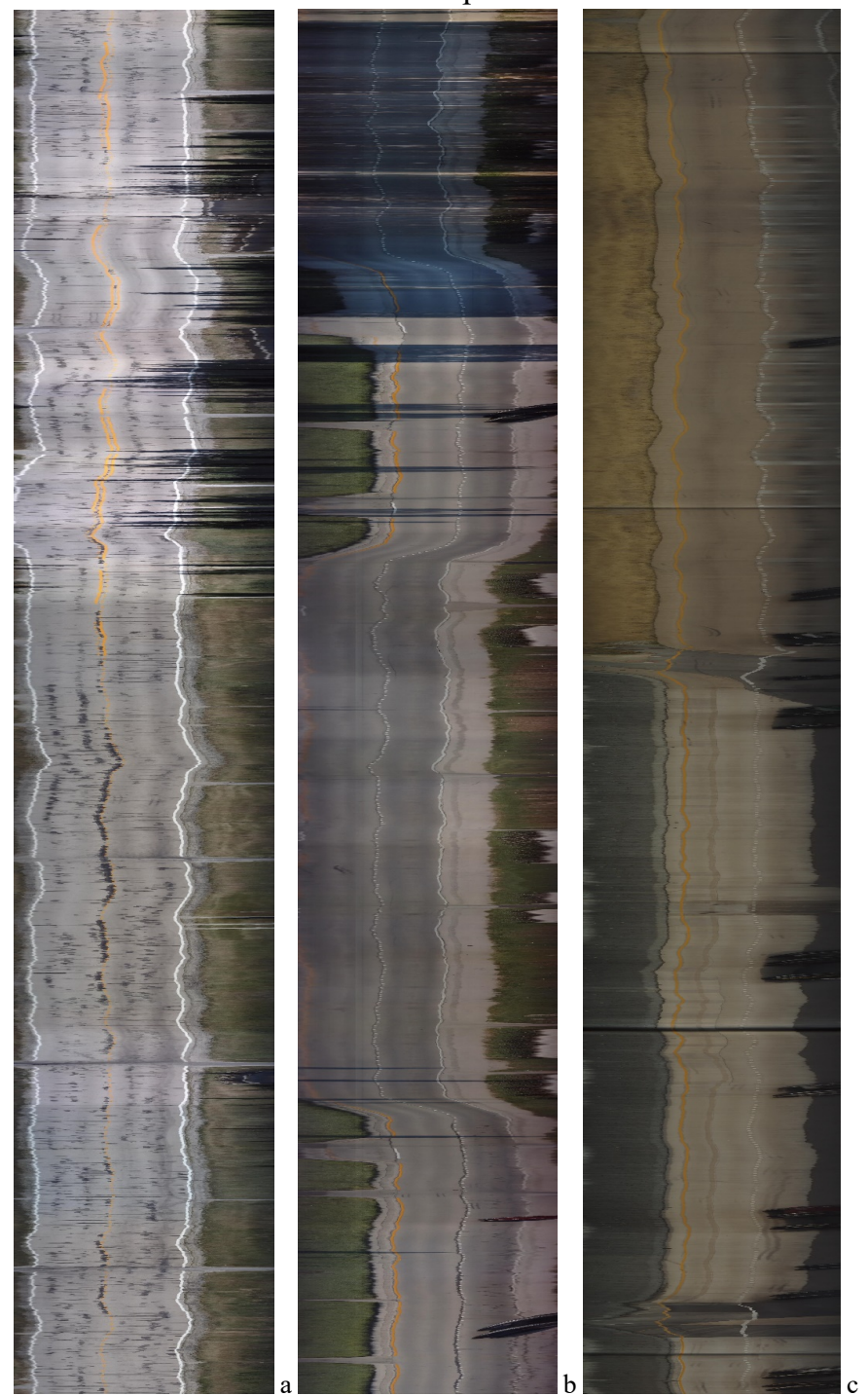

Fig. 2. Road profiles from 5-min HD video ( $9 \mathrm{k}$ positions at sampling rate 30 $\mathrm{fr} / \mathrm{sec}$ ) in different weathers. (a) Sunny day (b) Partly shadow (c) Cloudy.

The sampled pixel lines from consecutive frames are connected by their frame numbers to form a temporal road profile as in Fig. 2, which shows road and off-road regions about four lanes wide. Even if the vehicle is moving on a curved road, the road profile keeps road at center. The road edges and lanes are waved due to the waving of vehicle in pitch and roll on uneven roads. Lane changes of vehicles can also be observed in the profile. The vehicle stopping periods generate parallel stripes along the time axis (same scene repeats). They are excluded by a simple calculation. Detection of road edge position in the road profile can estimate its $3 \mathrm{D}$ position $15 \mathrm{~m}$ ahead and perform proper road departure avoidance. A vehicle passing at side lane may also leave a trace close to horizontal in the road profile as in Fig. 2b, but appears only in a short period.

The physical road edge is defined as the first position where material or elevation changes from road surface. The material changes such as asphalt-grass, asphalt-curb, asphalt- dirt, and so on cause differences in reflectance captured by the camera under sufficient illumination. For the illumination, we can briefly classify light sources to sunlight in sunny day, ambient light in cloudy day or in shadow, direct light from sun to camera, and head/street light in night. The elevation changes like curb or barrier also require sunlight or vehicle headlight to shade it. The reflection mode further includes diffused and specular reflections (or even highlight). The specular reflection appears on asphalt when facing the sun, and the highlight appears on wet roads.

The linearity at position $x$ on the sampling line, denoted as $l(x)$, is an accumulated number from lines through position $x$. Its value is roughly proportional to the line length. To estimate the road linearity, a belt of 30-pixel high is set around the sampling line. Within the belt, edge points are voted towards the sampling lines by using their gradient orientation. The linearity has strong response at a road edge, lane mark, curb, the side and bottom lines of passing, passed, or parked vehicles, as well as high-contrast texture on roadside as false positives. If the line is long and straight, the linearity is high. The linearity has low responses at local damage and noise spots, because of their short lengths. We consider the road edges mainly as slanted line in the video frames. We exclude vertical lines in the frames that are mostly from the side edges of surrounding vehicles, poles and buildings, and the reflection of objects and lights on wet roads. In a rainy day, reflection of scenes and vehicle lights on wet surface affects the road edge detection significantly.

In more details as in Fig. 3, an edge point with its gradient stronger than a threshold is detected at $(x, y)$ in the belt with edge orientation $T(x, y)$ from its gradient direction $\mathrm{G}_{\mathrm{x}} / \mathrm{G}_{\mathrm{y}}$, where $\mathrm{G}_{\mathrm{x}}$ and $\mathrm{G}_{\mathrm{y}}$ are horizontal and vertical filtering output with a differential operator. Extending the edge along $T(x, y)$ to the sampling line, the intersection is computed as $k$ at

$$
k=x+(y-h) \mathrm{G}_{\mathrm{y}} / \mathrm{G}_{\mathrm{x}} \quad \text { subject to }\left|G_{y}\right|>\delta
$$

where $h$ is the height of sampling line in the frame, and $\delta$ is a threshold to remove the influence from vertical lines. We accumulate the vote at $k$ by $l(k)=l(k)+1$ for linearity. We have also found that tiny texture, e.g., grass on roadside, has insufficient resolution for analysis. In addition, we store $G_{x}$ and $G_{y}$ as the homogeneity measure on road.

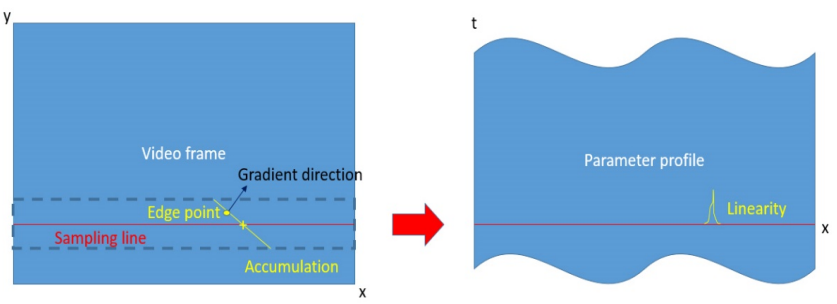

Fig. 3. Linearity estimation from an image frame to include structure information of road. (left) road edge and image belt in frame, (right) store linearity with the road profile in the same coordinate system.

To record weather/illumination, we further sample several image regions as in Fig. 1. Colors are averaged in the roadside regions between the horizon and sampling line, which are less dependent to individual roadside objects. Sky is also sampled directly to reflect the weather. These data are obtained from each frame and the data sequences from the clip are saved. 


\section{WEATHER AND ILLUMINATION UNDERSTANDING}

\section{A. Weather and Illumination Categories Tagged by Humans}

Illumination affects the road appearance significantly. We integrate seasons with surface materials, because seasonal changes such as snow-covered ground, yellow grass, and wet road actually alter the surface reflectance of roadsides. We also combine weather and time to describe the illumination in intensity and direction. The direction of sun light may cause the specular reflection on road surface. We further combine weather and camera sensitivity in the same property because snowy may block a certain percentage of light reflected from the ground. Heavy rain and dirty windshield have the same effect on the camera sensitivity. Table I tags weather and illumination into 10 categories [10] based on visual properties of road and off-road relation.

Table I Various weather and illumination conditions Sunny has two types according to reflection property. Sunny back-to-thesun (BS) of a camera includes side-to-the-sun. The camera receives diffused reflection of scenes in saturated color. It also includes partly cloudy, as a road may show sunny while the sky far away has cloud. Sunny facing-the-sun (FS) involves highlight or specular reflection on road surface, and shadow on most standing objects/scenes. Sunny FS generally has lower saturation than Sunny BS in the video frame.

Shadow indicates from place to place or a whole segment of route in the shadow of trees or buildings. It is like dark lit if the vehicle is in a deep forest with full of shadow. The intensity drops significantly in shadow. Cloudy is mainly overcast. It varies from bright sky to dark rainy cases. Dark lit refers to low illumination on road, where road edges are barely visible, while sky is still bright as ambient light. This usually happens in dusk after sun set or bad weather.

Dirty windshield includes dirty spots on glass and object reflection from dashboard to the camera, when the camera faces the sun in direct lighting. These patterns will disturb the visibility of road to a certain degree. Fog blurs the entire scenes on their sharpness and whitens the field of view by adding a layer of water particles [1].

Raining is the situation when water drop reduces the visibility. It is classified either wiper is on or windshield has raindrop on it [14]. Snowing is similar as raining while the visibility is reduced more. Roadside is probably being snow-covered more frequently than road surface. Night video mostly have dark sky but with street lights and vehicle head lights on. Wet ground at night has drastic reflection of lights.

These qualitative categories are associated with dry road. Some weathers such as raining, night, and snowing change the road appearance completely on wet and snow-covered roads. Moreover, the weather/illuminations tagged by humans have ambiguity and overlap between each other in the feature space. To use such information for road edge detection, we cluster them numerically using K-mean algorithm.

\section{B. Clustering and Classification of Weather and Illumination}

We apply data mining approach on a large set of driving videos captured over a year [13]. Various types of roads including urban, rural, residential roads and highway are scanned through all seasons [10]. To test the road edge detection results, we use the same driving video database.

For the clustering of weather/illuminations, 360 video clips are selected. Each is 5-min long so that the total yields $340 \mathrm{k}$ positions. We compute feature vector $\boldsymbol{y}$ in each frame with 9 variables such as intensity $I$ and chrome sat from the sky, roadside regions, and road surface, as well as their variation (var). Video frames are clustered by K-means algorithm, which results in clusters $S_{i}, i=1 \sim 7$, more stable and discriminable than human specified categories. Such equally divided clusters in the feature space also give their centroids [10]. In this paper, we intuitively give each of K-mean clusters with a close category name tagged by humans for easy understanding.
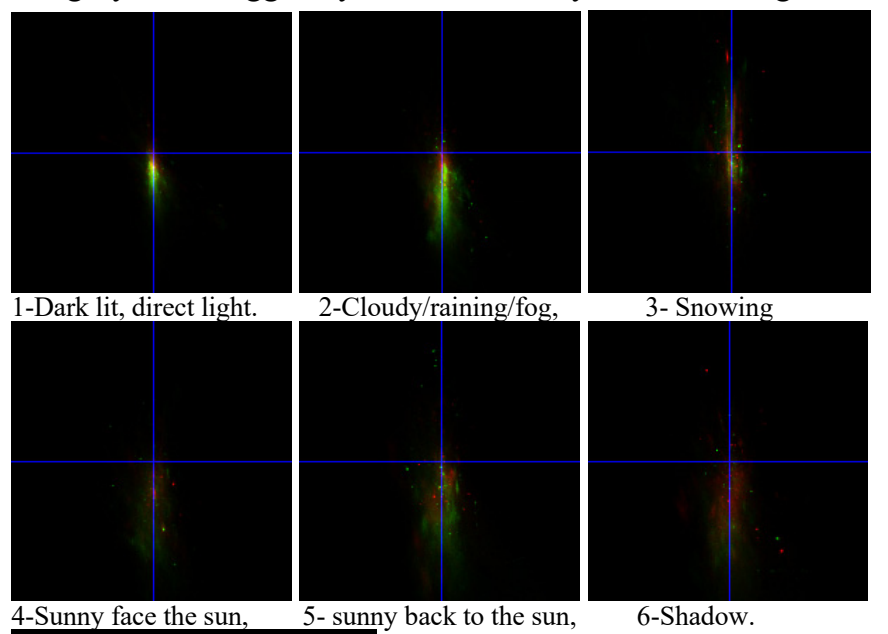

5- sunny back to the sun,

6-Shadow.

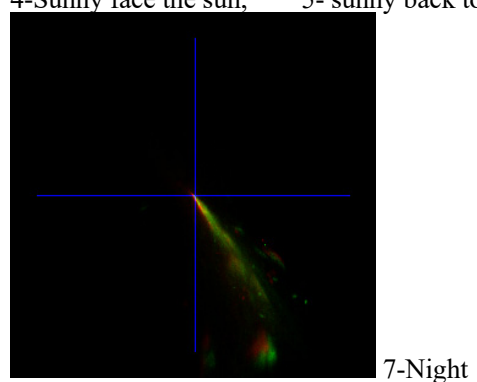

Every cluster is displayed in the Chroma and Intensity space (2D), normalized with the highest frequency. The vertical axes downward are the intensity difference, and the horizontal axes are the chroma difference. $\mathrm{R}$ channels are the left-side differences and $\mathrm{G}$ channels are the right-side differences.

Fig. 4. Distribution of color differences (road - off-road) at edges for 7 clusters.

The second phase after clustering is the classification of videos for weather based road edge detection. For a video frame in the database, we classify its weather/illumination by computing its distance from all cluster centroids in the feature space, and select the closest cluster as its class. This classification allows us to find the likelihood probability of on-road and off-road differences as shown in Fig. 4.

\section{RoAd EdGE IDENTIFICATION BASED ON Mined DATA}

\section{A. Feature Computation of Road Edge Candidates}

The detection of road edges is carried out in the road profile. We use the features of color, linearity, and homogeneity on road and off-road for identifying road edges $15 \mathrm{~m}$ ahead of vehicle, assuming the road edge is not blocked by other vehicles. Road edges are searched from the center, i.e., the vehicle heading direction, towards both sides. The road surface color is grasped from the heading direction.

Assume lane tracking works separately on good road as a redundant function to prevent lane departure. We apply median filter on Chroma and Intensity in the road profile to remove the lane marks so that our decision does not rely on lane marks. This operation also removes the small portholes captured by edges. Above that, edge detection is applied horizontally in the resulted road profile. We pick up edges higher than a proper threshold to erase spots of small damages. Linearity of road has been calculated from the frame 


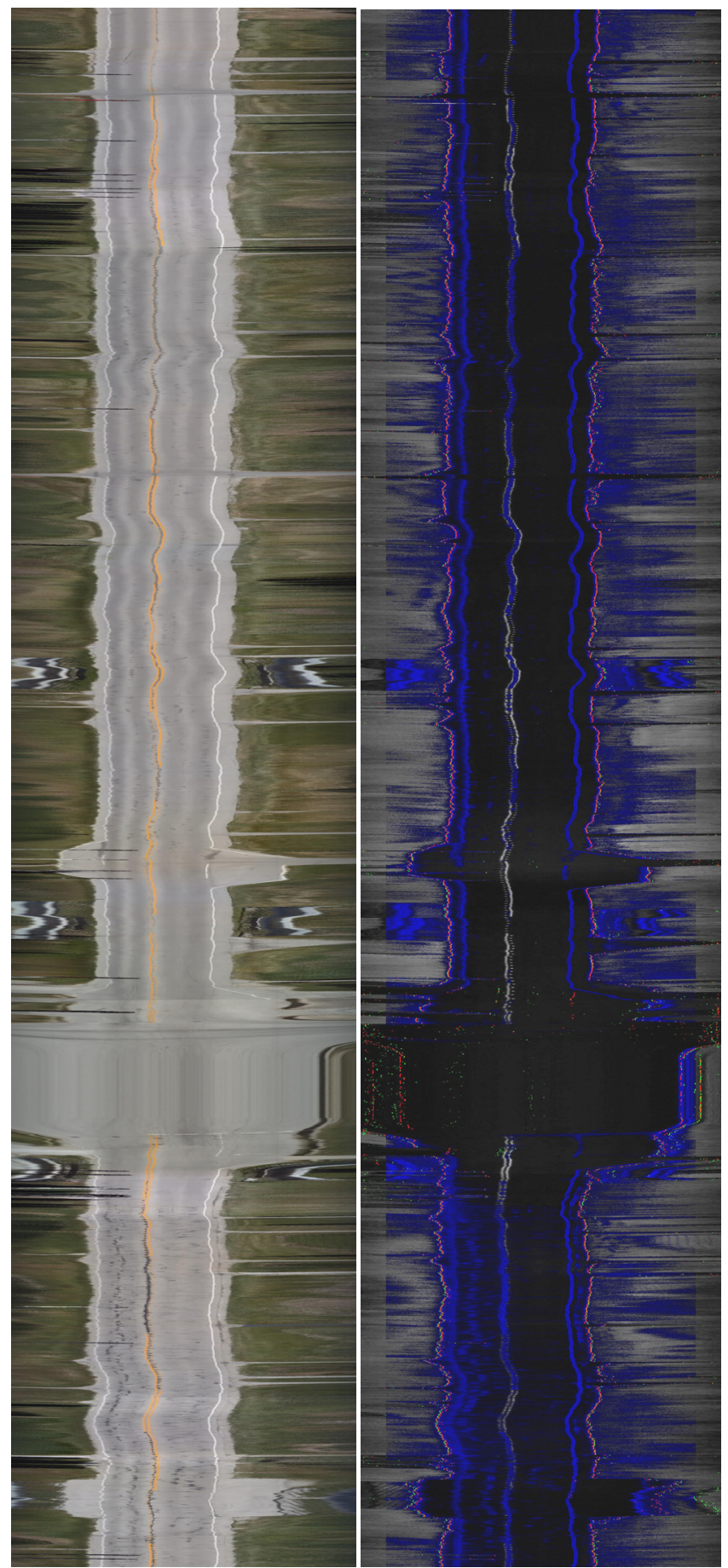

Fig. 5. Five minute road profile in a sunny day (left) and computed feature outputs (right) of chroma (in gray area), overwriten by the mixture of linearity (blue), the highest chroma changes (green points), and the highest intensity changes (red points) on two sides of road.

to verify the edge candidates. By examining videos and road profiles of each weather/illumination clusters, we visualize the intensity and saturation after horizontal median filtering and linearity extraction in road profiles as shown in Fig. 5. We can observe that the method can locate road edges near perfectly under good illumination conditions.

Up to this stage, our method is similar as other methods. However, our road edge detection performs differently when the road edge has many uncertain candidates under weak illuminations. The methods proposed so far focused on optimization to overcome the uncertainty in an ad-hoc approach; no solid evidence from real data provided. We examine the discriminative features under different weathers, and use the learned data model to confirm the road edge decision. Qualitatively, we visualize the feature responses in the road profiles for all clusters and examine their effectiveness in locating road edges. A feature ranking is summarized in Table II based on the observed results. Intensity contrast $\Delta I$, chroma difference $\Delta s$ are the most reliable features in order accompanied with linearity $L$.

\section{B. Bayesian Method for Road Edge Detection}

Quantitatively, our road edge detector further uses Bayesian method that benefits from the data clustering. A minimum threshold is set to accept non-horizontal edges in the road profile that may divide on-road and off-road regions. For multiple edges at $x_{n}, n=1,2,3, \ldots$ on each side from center as illustrated in Fig. 6, we pick one final road edge with the most proper difference value on its sides fitting with what have been learned in data mining stage. The strongest difference of intensity or chorma does not necessary imply a correct road edge. For example, the reflection of vehicle rear lights on wet roads is much stronger than real road edges, but is incorrect.

Within the weather/illumination clusters, we investigate visual properties around road edges. Because road surfaces are mostly asphalt that have black-to-gray color and thus low chroma value, the color difference $\boldsymbol{d}$ (onroad-offroad) in chroma and intensity space between road and roadside regions are computed from each frame in the video set. The distribution of $\boldsymbol{d}$ is obtained for each cluster as in Fig. 4; $\boldsymbol{d} i$ for cluster $i$ is plotted in RGB channels to indicate left and right differences. This serves as likelihood probability $P\left(\boldsymbol{d}_{i} \mid \mathrm{E}, \mathrm{S}_{\mathrm{i}}\right)$ $i=1 \sim 7$, where $\mathrm{E}$ is the road edge. In such distribution, we can observe several spots having high values in Fig. 4, which are related to special materials on road sides. Because a road surface has low chroma value, the difference in chroma is almost positive for all clusters except snow and dark lit. The intensity differences are mostly negative for all clusters except snow-covered roadside and new asphalt road surface, because the road surface is generally brighter than roadside materials.

In each frame, the difference across each edge candidate at $x_{n}$ can be described by the contrasts in intensity and chroma, as well as linearity, as $d_{n}=\{\Delta I, \Delta s, L \ldots\}$ computed from horizontal differential filter on the median filtered results. After weather/illumination class $S_{i}$ is identified, the likelihood probability of difference $\mathrm{P}\left(d_{n} \mid \mathrm{E}, S_{i}\right)$ is employed to identify the road edge in the frame. For multiple candidates at $x_{n} n=1,2,3$, with differences $d_{n}$ on each side, we find one road edge with the maximum probability $\mathrm{P}\left(E \mid d_{n}\right)$ among all $n$, where road edge $E$ is unique on one side of road, that is

$$
x_{n}=\arg \max _{\mathrm{n}}\left\{\mathrm{P}\left(\mathrm{E} \mid d_{n}\right)\right\}
$$

Using the Bayesian rule, $\mathrm{P}\left(\mathrm{E} \mid d_{n}\right)$ is calculated as

$$
\mathrm{P}\left(\mathrm{E} \mid d_{n}\right)=\mathrm{P}\left(\mathrm{E}, d_{n}\right) / \mathrm{P}\left(d_{n}\right)=\mathrm{P}\left(d_{n} \mid \mathrm{E}\right) \mathrm{P}(\mathrm{E}) / \mathrm{P}\left(d_{n}\right)
$$

where prior probability $\mathrm{P}(\mathrm{E})$ is constant 0 for invisible road edge of a wide road, and 1 for visible road edge in the frame. $\mathrm{P}(\mathrm{E})$ is common for all edge candidates in the frame. The 
Table II Qualitative feature selection via data mining. Effective features at edge are listed in order. $\Delta \mathrm{I}$ : intensity contrast (inner side - outer side); $\Delta \mathrm{s}$ : chroma/saturation contrast; $\mathrm{S}$ : chroma/ saturation; L: linearity; $\mathrm{G}_{\mathrm{x}}$ : difference (gradient) across road edge; $\mathrm{G}_{\mathrm{y}}$ : difference along road; R: larger region after media filtering; $\mathrm{H}$ : hue.

\begin{tabular}{|c|c|c|c|c|c|c|c|c|}
\hline On-road & \multicolumn{7}{|c|}{ Dry road } & Wet road \\
\hline $\begin{array}{l}\text { Roadside } \\
\text { materials }\end{array}$ & $\begin{array}{l}\text { Grass, } \\
\text { Vege }\end{array}$ & $\begin{array}{l}\text { Tree, } \\
\text { forest }\end{array}$ & $\begin{array}{l}\text { Gravel, } \\
\text { dirt }\end{array}$ & $\begin{array}{l}\text { Curb, } \\
\text { concrete }\end{array}$ & $\begin{array}{l}\text { Rail, } \\
\text { barrier }\end{array}$ & Cone & $\begin{array}{l}\text { Snow- } \\
\text { covered }\end{array}$ & All \\
\hline Night & \multicolumn{3}{|c|}{$\begin{array}{l}\text { Edge invisible without lane } \\
\text { mark, } \\
\text { Have to use L }\end{array}$} & $\begin{array}{l}\text { L, if edge } \\
\text { in head } \\
\text { light }\end{array}$ & $\Delta \mathrm{I}, \mathrm{L}$ & \multirow{10}{*}{$\mathrm{H}, \mathrm{S}$} & \multirow{10}{*}{$\Delta \mathrm{I}<0$} & $\begin{array}{l}\text { Low } \mathrm{G}_{\mathrm{x}} \\
\left|\mathrm{G}_{\mathrm{y}}\right| \neq 0, \mathrm{~L}\end{array}$ \\
\hline Dark lit & \multicolumn{5}{|c|}{$\Delta \mathrm{I}$ (low) on R (detectable) } & & & $\mathrm{L}$ \\
\hline Direct light & \multicolumn{5}{|c|}{$\Delta \mathrm{I}$ (low), mostly failed } & & & $\mathrm{L}$ \\
\hline Rainy & \multicolumn{5}{|c|}{$\Delta \mathrm{I}$, confirmed by $\mathrm{L}$} & & & $\mathrm{L},\left|\mathrm{G}_{\mathrm{y}}\right| \neq 0$ \\
\hline Cloudy & $\begin{array}{l}\Delta \mathrm{I}, \Delta \mathrm{s}, \\
\mathrm{L}\end{array}$ & $\begin{array}{l}\Delta \mathrm{I}, \Delta \mathrm{s}, \mathrm{L}, \\
\mathrm{G}_{\mathrm{x}}\end{array}$ & \multicolumn{3}{|c|}{$\Delta \mathrm{I}, \mathrm{L}$} & & & $\begin{array}{l}\Delta \mathrm{I}, \Delta \mathrm{s}, \mathrm{L}, \\
\left|\mathrm{G}_{\mathrm{y}}\right| \neq 0\end{array}$ \\
\hline Fogy & \multicolumn{5}{|c|}{$\Delta \mathrm{I}$} & & & \multirow{2}{*}{$\begin{array}{l}\text { Less } \\
\text { possible } \\
\text { cases }\end{array}$} \\
\hline Snowing & \multicolumn{5}{|c|}{$\Delta \mathrm{I}$} & & & \\
\hline Sunny FS & \multicolumn{5}{|c|}{$\Delta \mathrm{I}, \Delta \mathrm{s}, \mathrm{L}$} & & & \multirow{3}{*}{$\begin{array}{l}\text { Not } \\
\text { existing } \\
\text { cases }\end{array}$} \\
\hline Shadow & $\begin{array}{l}\mathrm{L}, \Delta \mathrm{s}, \\
\mathrm{H}, \mathrm{G}_{\mathrm{y}}\end{array}$ & $\Delta \mathrm{I}, \mathrm{H}, \mathrm{G}_{\mathrm{x}}$ & $\Delta \mathrm{I}$ & $\mathrm{L}$ & $\mathrm{L}$ & & & \\
\hline Sunny $B S$ & $\begin{array}{l}\mathrm{R}, \Delta \mathrm{I}>0 \\
\Delta \mathrm{s}<0\end{array}$ & $\begin{array}{l}\mathrm{R}, \Delta \mathrm{I}>0 \\
\Delta \mathrm{s}<0, \mathrm{G}_{\mathrm{x}}\end{array}$ & $\Delta \mathrm{I}$ & \multicolumn{2}{|l|}{$\Delta \mathrm{I}, \mathrm{L}$} & & & \\
\hline
\end{tabular}

likelihood probability $\mathrm{P}\left(d_{n} \mid \mathrm{E}\right)$ has been obtained in Fig. 4. Probability $\mathrm{P}\left(d_{n}\right)$ is accumulated for the entire video clip since it is related to the exposure of cameras for long driving.

\section{EXPERIMENTS}

The experiments on road edge detection have been carried out on 150 randomly selected road profiles (each 9k frames) along with the extracted sequences of weather, linearity, and homogeneity. Their weather/illumination is classified first so that we are able to select features in road edge detection. The weather classification has reached a good accuracy [10] so that we can apply the maximum likelihood to the limited number of candidates by (3) for road edges. The detection accuracies of road edges are given in Table III. We count the frame numbers when the road edges are located correctly among the entire length of road profiles. Each weather/illumination cluster is tested at $27 \mathrm{k}$ frames in the profiles.

TABLE III ACCURACY OF ROAD EDGE DETECTION

\begin{tabular}{|l|l|l|l|l|l|}
\hline $\begin{array}{l}\text { WEATHER/ } \\
\text { ILLUMIATIONS }\end{array}$ & NIGHT & DARK LIT & $\begin{array}{l}\text { DIRECT } \\
\text { LIGHT }\end{array}$ & RAINY & CLOUDY \\
\hline ACCURACY & $90.58 \%$ & $81 \%$ & $63.63 \%$ & $54.36 \%$ & $88.04 \%$ \\
\hline & FOGY & SNOWY & SUNNY FS & SHADOW & SUNNY BS \\
\hline & $84.53 \%$ & $79.42 \%$ & $91.08 \%$ & $70 \%$ & $87.03 \%$ \\
\hline
\end{tabular}

Sunny and cloudy: road edges are detected correctly under the bright illuminations (Fig. 7), which is not much different from what other works have achieved. Figure 8 shows the curb detection based on shadow/shading on concrete surfaces.
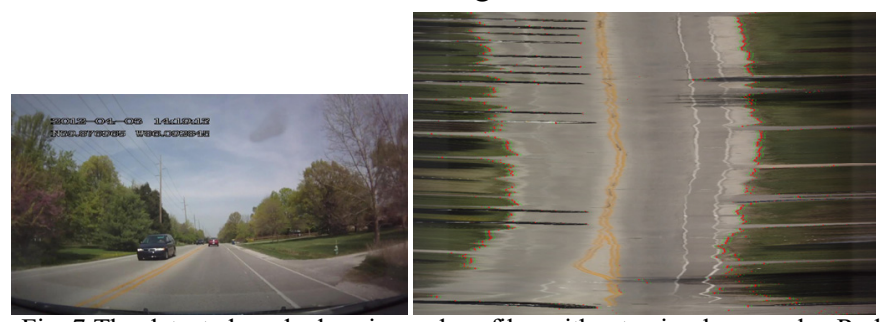

Fig. 7 The detected road edges in road profiles without using lane marks. Red bounded by two side green are detected road edges. High accuracy results in output outside road shoulder. Except some passing vehicles bother the edge with close-to-horizontal traces, the road edges are located almost perfectly.
Dark road surfaces: for raining, shadow, and dark lit, the accuracies are much lower. Road edges under dark lit can still be detected correctly because there is less interference from bright sky as shown in Fig. 9. On the other hand, direct light has brighter edges than road edges. Light from the sun causes unstable intensity, windshield patterns, and highlight on road. The detection accuracy drops significantly.

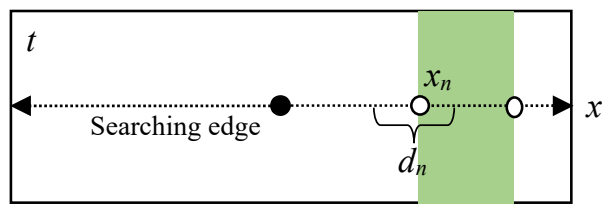

Fig. 6 Road profile for the road edge selection from multiple edge candidates. Feature properties on two sides of edge candidates are considered as evidence.

Night and invisible roadside: Because of the limited scope lit by vehicle headlight, road edge in dark is unable to be located if road is wide (Fig. 10). We count such an edge safe since it does not reach the real edge at wide end, unless road edge is missed when it is in the scope of headlight. Wet urban roads in night is a problem since traffic and street lights have long reflection on road, which causes much confusion.

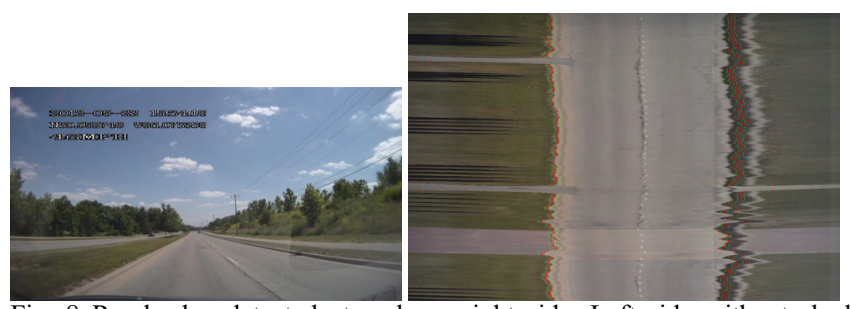

Fig. 8 Road edge detected at curb on right side. Left side without shadow causes the road edge detected at grass margin.
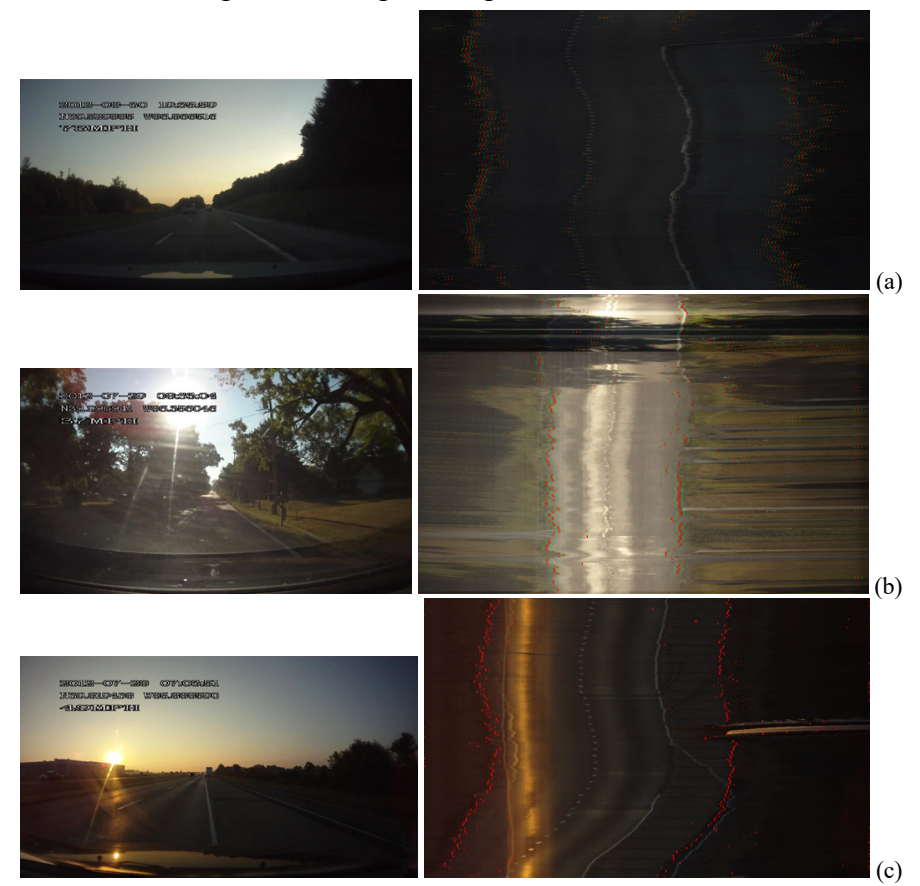

Fig. 9. Dark roads. (a) Dark lit roadside is barely visible from intensity changes. The detected positions out of three lanes are marked correctly in red. $(b, c)$ 
Direct light and detected road edge marked in red. (b) is closer to Sunny facing the sun with shadow of tree. (c) is closer to dark lit case.
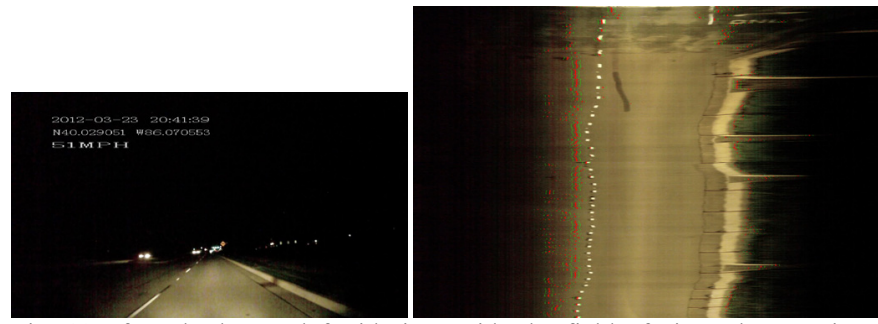

Fig. 10. If road edge on left side is outside the field of view, the margin of headlight lit region is marked as road edge. If a road edge is visible in a close position as right, it can be detected correctly. The algorithm only detects close road edge within the lit region, but not reaches far side.

Raining and wet road: rain has a low illumination like cloudy. If road is less wet, the algorithm performs correctly as cloudy case (Fig. 11(a)). When a road accumulates much water, many vertial edges are reflected in the road area from roadside buildings, poles, street lights, as well as vehicle breaking lights and headlights. Highway with no high buildings in background is less affaceted by reflectoin. Our linearity computation from non-vertical lines reduces such influences. However, the most annoying case is the wet urban road in night as in 11(b), where real road edge is weak in intensity and then in linearity.

Sunny facing the sun: the illumination makes bright road surface and background scenes in shadow. The chroma is small because of the gray-to-white road surface and shadow on objects. The intensity change is relatively large in such a case as shown in Fig. 12.
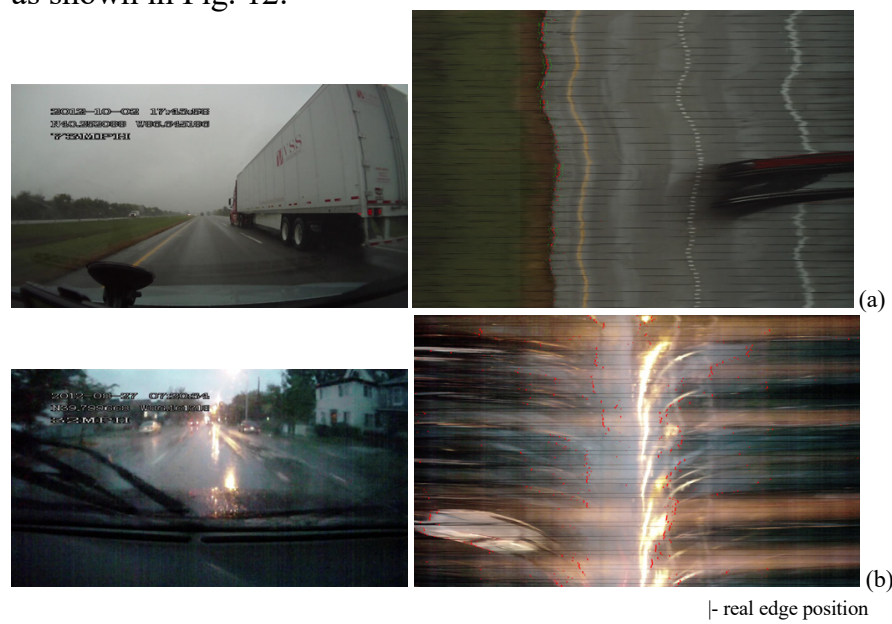
Fig. 11 Raining weather. (a) Road is not very wet yet. Wiper motion is visible
as horizontal black lines in the profiles but has little influence on the results. (b) Raining on wet road with light reflection in night. Detected edge are wrong.
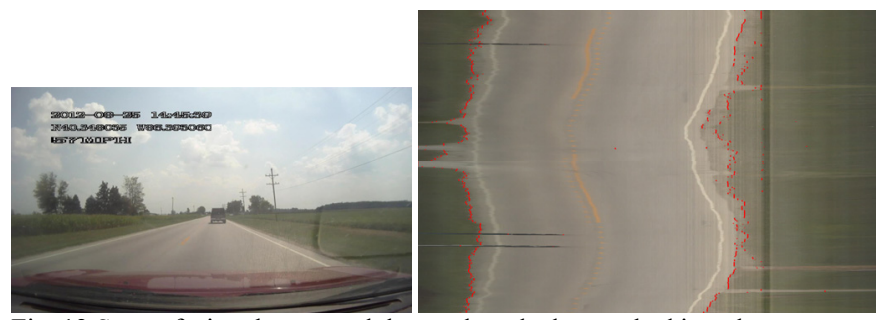

Fig. 12 Sunny facing the sun and detected road edge marked in red.

Snowing and snow-covered roadside: we found the intensity on-road lower than off-road in data mining, because roadsides always get snow covered earlier than road surface (Fig. 13).
The accuracy of snow result is lower than sunny even if it has bright environment, because of some snow remains on road surface. Linearity is not always available if snow has not melt.

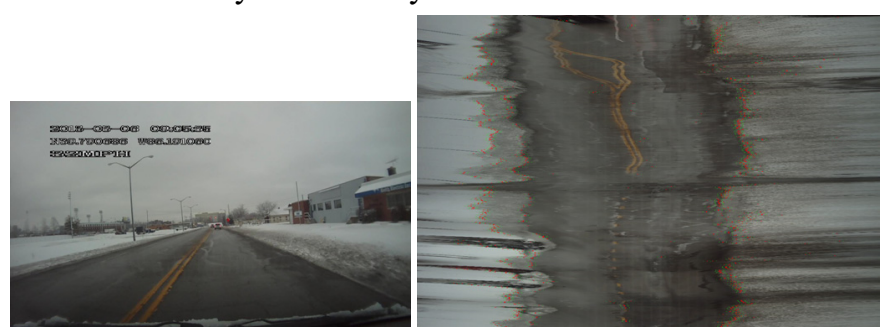

Fig. 13 Snow-covered edges have poor linerity, but are brighter than drive way.

\section{CONCLUSION}

This paper tackles the road edge detection by vehicle borne video to prevent road departure. We develop algorithms based on data mining results from large sets of naturalistic driving video. The weather and illumination conditions of a video is first identified. Based on the machine learned likelihood of the road edges in such conditions, the road edges are extracted in the road profile images from driving videos. This reduces the error of road edges due to noises such as highlight, wet road reflection, shadow and so on. The data mining gives evidence to detect various edges from grass to curb under whole the spectrum of illumination conditions.

\section{REFERENCES}

[1] N. Gimonet, A. Cord, G. Saint Pierre, "How to predict real road state from vehicle embedded camera?" IEEE IV 2015, 593-598.

[2] F. Oniga, S. Nedevschi, "Processing dense stereo data using elevation maps: road Surface, traffic isle, and obstacle detection", IEEE Trans. on Vehicular Technology, Vol. 59, No. 3, 2010.

[3] H. Kong, J. Audibert, and J. Ponce, "Vanishing point detection for road detection," in Proc. IEEE CVPR., Jun. 2009, pp. 93-103.

[4] L. Nguyen, S. L. Phung, and A. Bouzerdoum, "Efficient vanishing point estimation for unstructured road scenes," 2016 Int. Conf. on Digital Image Computing: Techniques and Applications (DICTA), Nov. 2016.

[5] J. Huang, B. Kong, B. Li, and F. Zheng, "A new method of unstructured road detection based on HSV color space and road features," International Conference on Information Acquisition, Jul. 2007.

[6] S. Yun, Z. Guo-ying, and Y. Yong, "A road detection algorithm by boosting using feature combination," IEEE IV, Jun. 2007.

[7] T. Veit, J.-P. Tarel, P. Nicolle, and P. Charbonnier, "Evaluation of road marking feature extraction," 11th IEEE ITSC, Oct. 2008.

[8] Guo, S. Mita, "Semantic-based road environment recognition in mixed traffic for intelligent vehicles and advanced driver assistance systems", 15th Int. IEEE ITSC, 444-450, 2012.

[9] C. Fern'andez, D. F. Llorca, C. Stiller, M. A. Sotelo, "Curvature-based curb detection method in urban environments using stereo and laser", IEEE IV 2015, 579-584, 2015

[10] G. Cheng, Z. Wang, J.Y. Zheng, "Big-video mining of road appearances under full spectrums of weather and illuminations", IEEE ITSC 2017.

[11] M. Kilicarslan, J. Y. Zheng, "Temporal video profile from driving video," IEEE IV, pp. 529-551, 2014

[12] J. Zhang, S. Xia, K. Lu, H. Pan, A. K. Qin, "Robust road detection from a single image", 23th ICPR 2016, 854-859

[13] R. Tian, L. Li, K. Yang, S. Chien, Y. Chen, R. Sherony, "Estimation of the vehicle-pedestrian encounter/conflict risk on the road based on TASI 110-car naturalistic driving data collection. IEEE IV 2014, 623-629.

[14] H. Kurihata, T. Takahashi, I. Ide, Y. Mekata, H. Murase, Y. Tamatsu, T. Miyahara, "Rainy weather recognition from in-vehicle camera image for driver assistance", IEEE IV2005.

[15] X. Yan, et. al, "Weather recognition based on images captured by vision system in vehicle", Advances in Neural Networks, 2009.

[16] A. Gem, R. Moebus, U. Franke, "Vision-based lane recognition under adverse weather conditions using optical flow", IEEE IV 2002. 